\title{
Efeitos do tratamento com formalina e sulfato de cobre sobre os parâmetros hematológicos e parasitos monogenéticos em juvenis de Hemigrammus sp. (Osteichthyes: Characidae)
}

\author{
Luciana Farias PAIXÃO ${ }^{1,4}$, Rudã Fernandes Brandão SANTOS ${ }^{3,5}$, Fabricio Menezes RAMOS ${ }^{1,6}$, \\ Rodrigo Yudi FUJIMOTO ${ }^{2,7}$
}

\begin{abstract}
RESUMO
O objetivo deste trabalho foi avaliar a eficácia da formalina e sulfato de cobre no controle de monogenéticos em juvenis de Hemigrammus sp e seu efeito na contagem diferencial de leucócitos. Utilizou-se um delineamento inteiramente casualizado com 5 tratamentos e 3 repetiçóes. Todos os tratamentos tiveram duração de três dias e corresponderam a: T1, controle; T2

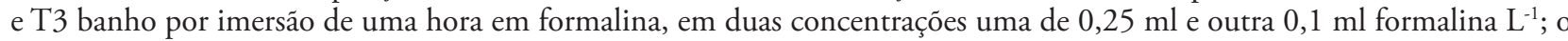
T4, banho de 24 horas com $0,025 \mathrm{ml}$ de formalina $\mathrm{L}^{-1}$; e o T5, banho de 24 horas com sulfato de cobre $\left(0,3 \mathrm{mg} \mathrm{L}^{-1}\right)$. Após o período experimental observou-se que em T2 ocorreu 100\% de mortalidade. O T3 e T5 proporcionaram eficácia de $100 \%$, porém os peixes apresentaram intoxicação e mortalidades de 66 e $80 \%$, respectivamente. T4 apresentou eficácia de 77,7\% e diminuição dos índices parasitológicos: número total de parasitos de $11,3 \pm 9$, intensidade média de infecção de 3,2 2 e prevalência de 47,1\%. Quanto aos leucócitos observou-se que no T3, T4 e T5 ocorreram diferenças na proporção de linfócitos, monócitos e neutrófilos em relação ao controle. $\mathrm{O} \mathrm{CuSO}_{4}$ e a formalina reduzem a carga parasitária, mas apresentaram-se tóxicos nas maiores concentrações.
\end{abstract}

PALAVRAS-Chave: $\mathrm{CuSO}_{4}$, formol, monogênias, tratamento

\section{Evaluation of formalin and copper sulfate on monogenetic parasite (Urocleidoides sp.) of Hemigrammus sp. (Osteichthyes: Characidae) juveniles}

\begin{abstract}
The objective of this study was to evaluate the effectiveness of formalin and copper sulphate to control monogeneans in juvenile Hemigrammus sp and its effect on leukocyte count. We used a completely randomized design with five treatments and three replications. All treatments lasted for three days and consisted of: T1, control; T2 and T3 immersion bath for one hour in formalin, an of two concentrations of $0.25 \mathrm{ml}$ formalin and the other $0.1 \mathrm{ml} \mathrm{L}^{-1}$; the T4 bath for 24 hours with $0,025 \mathrm{ml}$ of formalin- $\mathrm{L}^{-1}$; and T5, bath 24 hours with copper sulphate $\left(0.3 \mathrm{mg} \mathrm{L}^{-1}\right)$. After the experimental period was observed that T2, $100 \%$ mortality. The T3 and T5 provided 100\% efficiency, however, fish presented mortalities and intoxication of the 66 and $80 \%$ respectively. T4 showed an efficacy of $77.7 \%$ and decrease in parasitological indices: total number of parasites of 11,3 \pm 9 , mean infection intensity of $3.2 \pm 2$ and a prevalence of $47.1 \%$. As the leukocytes was observed that at T3, T4 and T5 occurred differences in the proportion of lymphocytes, monocytes and neutrophils as compared to control. The $\mathrm{CuSO}_{4}$ and formalin reduce the parasite load, but presented to be toxic at higher concentrations.
\end{abstract}

KEYWORDS: control, $\mathrm{CuSO}_{4}$, formalin, monogenea

\footnotetext{
1 Universidade Federal do Pará, Campus de Bragança. Alameda Leandro Ribeiro, s/n bairro aldeia, Bragança Pará Cep 68600-000 PA-BR

2 EMBRAPA, Tabuleiros Costeiros Av. Beira Mar, 3250 - Jardins Caixa Postal 44 - Aracaju, SE - Brasil -, Cep 49025-040

${ }^{3}$ Universidade Estadual Paulista, Júlio de Mesquita Filho. Via de Acesso Prof. Paulo Donato Castellane, s/n - Jaboticabal, SP - Brasil - Cep 14884-900;

${ }^{4}$ Formada em Ciências biológicas pela Universidade Federal do Pará;

${ }^{5}$ Mestrando pelo programa de Pós Graduação em Aquicultura pela UNESP, CAUNESP

${ }^{6}$ Doutorando pelo programa de Pós Graduação em Ciência Animal pela Universidade Federal do Pará;

7 Pesquisador EMBRAPA Tabuleiros Costeiros.
} 


\section{INTRODUÇÃO}

O gênero Hemigrammus é amplamente distribuído no Brasil, sendo encontrado geralmente em riachos e apresentando habito alimentar onívoro (Casatti et al. 2003; Brandão-Gonsalvez et al. 2010). Na Amazônia diversas espécies desse gênero tem importância econômica como $o H$. armstrongi, H. bleheri, H. erithrozonus, $H$. ocellifer, H. ulreyi devido suas características propicias para serem utilizados na aquariofilia.

A cadeia de comercialização dessas espécies apresenta alguns gargalos, iniciando na fase de captura em que os peixes são mantidos em basquetas plásticas nos barcos de pescadores sem trocas de água e com alimentação inadequada, após esse período os peixes sofrem problemas relacionados com transporte e qualidade de água nesses recipientes e posteriormente já nos entrepostos comerciais onde comumente são estocados antes da exportação são armazenados em densidades elevadas e recebem tratamentos preventivos inadequados (Torres et al. 2008).

Como esses manejos geram estresse nos peixes e consequente diminuição da resistência, muitas vezes ocorre o aparecimento de surtos de doenças e parasitoses resultando no desequilíbrio da tríade parasito-hospedeiro.

Assim, infestaçóes por parasitas monogenéticos caracterizados por serem monoxêmicos (Garcia et al. 2003) e apresentar infestaçôes que resultam em lesôes que são portas de entrada para bactérias e fungos tem potencial para gerar grandes perdas econômicas. Todavia, pouco se sabe sobre as relaçôes entre os helmintos monogenóides e peixes ornamentais (Garcia et al. 2003).

Quando se considera os principais produtos químicos utilizados no controle e prevençấo da reproduçấo desses parasitos merece destaque a formalina e o sulfato de cobre (Martins 2004; Tavares-Dias et al. 2002). Podendo ser aplicado nas formas de banho, de longa ou de curta duração. Porém, para peixes tropicais a informação da duração destes banhos ainda é escassa podendo ser citado Araujo et al. (2004).

Estudos demonstraram efeitos tóxicos do formol em peixes, como agressão do epitélio branquial (Martins 2004), também há relatos que a toxicidade aumenta com a elevaçấo da temperatura da água (Carraschi et al. 2011) sendo esta afirmação muito importante para a realização de tratamentos em peixes tropicais. Para o sulfato de cobre é reportado que o mesmo é tóxico em água com baixa dureza e seu uso prolongado pode deixar os peixes debilitados (Bassleer 2011) além de que no ambiente natural já ocorrem problemas relacionados com o aumento da sua concentraçáo nos corpos de água ocasionando efeitos tóxicos nos organismos aquáticos (Mazon et al. 2002).
Diante desse quadro, a utilizaçáo de uma ferramenta que possibilite diagnosticar a higidez dos peixes durante a realização dos tratamentos preventivos com essas substâncias é importante para avaliar o uso adequado destes produtos. Assim, o monitoramento das respostas hematológicas se apresenta como uma alternativa, pois, Jung et al. (2003) afirmaram que os valores sanguíneos demostram a toxicidade de um produto químico. Porém, não existe uma padronização, para os peixes tropicais, do quadro hematológico o que dificulta a interpretação de resultados.

O objetivo deste trabalho foi avaliar a infestação parasitária de monogenéticos branquiais e contagem diferencial de leucócitos do peixe ornamental Hemigrammus sp. submetidos a diferentes tratamentos com formalina $(37 \%)$ e sulfato de cobre pentahidratado $\left(\mathrm{CuSO}_{4} 5 \mathrm{H}_{2} 0\right)$ como subsídio para manejo de exportação de peixes ornamentais.

\section{MATERIAL E MÉTODOS}

O Experimento foi realizado na Universidade Federal do Pará no campus de Bragança, no Laboratório de Ictioparasitologia e Piscicultura da UFPA. Foram coletados 200 peixes jovens Hemigrammus sp, provenientes do Rio Chumucuí, Bragança, Pará, Brasil (0105'50”S e 4647’33”W) transportados até o laboratório (6 km do local de coleta) e distribuídos em duas caixas de 500 litros, onde permaneceram em aclimatação durante 10 dias. Nas caixas havia aeração constante e a troca de $30 \%$ da água foi realizada todos os dias. Os peixes foram alimentados com ração comercial extrusada Poytara com pellets de $2 \mathrm{~mm}$ (28 PB) todos os dias de manha e a tarde.

Após o período de aclimatação os peixes foram distribuídos em 15 recipientes contendo 3 litros de água, na densidade de 5 peixes por recipiente (peso vivo médio de $0,4 \mathrm{~g}$ ), com aeração constante. $\mathrm{O}$ experimento foi conduzido $\mathrm{em}$ um sistema estático. Durante o experimento os peixes não foram alimentados e foram registradas as mortalidades, sendo realizada no fim do experimento a determinaçáo da taxa de mortalidade. Os parâmetros de água como o $\mathrm{pH}$, temperatura (YSI 60) e oxigênio dissolvido (YSI 550A) foram monitorados diariamente).

O delineamento utilizado foi inteiramente casualizado com 5 tratamentos e três repetiçóes. Os tratamentos foram denominados de: T1, tratamento controle; T2, banho com duração de uma hora na concentração de $0,25 \mathrm{ml}$ de formalina $\mathrm{L}^{-1}$ de água, repetido diariamente, por três dias; $\mathrm{T} 3$, banhos com duraçáo de uma hora na concentraçáo de $0,1 \mathrm{ml}$ de formalina $\mathrm{L}^{-1}$, repetido diariamente, por 3 dias; $\mathrm{T} 4$, banhos de 24 horas na concentraçáo de $0,025 \mathrm{ml}$ de formalina $\mathrm{L}^{-1}$ de água por 3 dias e T5, banho de 24 horas com soluçáo de sulfato de cobre na concentração de $0,3 \mathrm{mg} \mathrm{L}^{-1}$ por 3 dias. A cada término de banho, a água era retirada e renovada com água 
sem produtos, sendo que no controle o mesmo procedimento foi realizado Apresentar a composição e fórmula química da formalina $\left(37 \%, \mathrm{H}_{2} \mathrm{CO}\right)$ e sulfato de cobre pentahidratado $\left(\mathrm{CuSO}_{4} 5 \mathrm{H}_{2} \mathrm{O}\right)$.

Após o término do experimento, uma amostra de 33\% dos peixes de cada tratamento foi submetida a contagem de parasitos e a obtenção do sangue. Os peixes foram sacrificados por comoçáo cerebral e o sangue, devido ao pequeno tamanho dos peixes, foi coletado por corte da nadadeira caudal para confecção da extensão sanguínea seguido de coloração com Rosenfeld para a contagem diferencial de leucócitos, contudo, devido ao pequeno tamanho dos peixes e à quantidade insuficiente de sangue colhido, somente foi possível avaliar a proporção percentual dos diferentes tipos de leucócitos circulantes pelo método indireto em extensão sanguínea .

Para a análise parasitológica após essa etapa as brânquias foram retiradas, separou-se os arcos para observá-los entre lâmina-lâmina e então contar os monogenéticos in loco e posteriormente preservá-los em formol $10 \%$.

Após a obtençáo do número de parasitos por peixe, foram calculados a eficácia dos produtos testados [(número de parasitos no tratamento/número de parasitos no controle) $\mathrm{x} 100$ ] e os índices parasitológicos de prevalência [(número de peixes infectados/número de peixes examinados) x 100)] e intensidade média (número total de parasitos/ número de peixes parasitados), segundo Bush et al. (1997).

total de parasitos

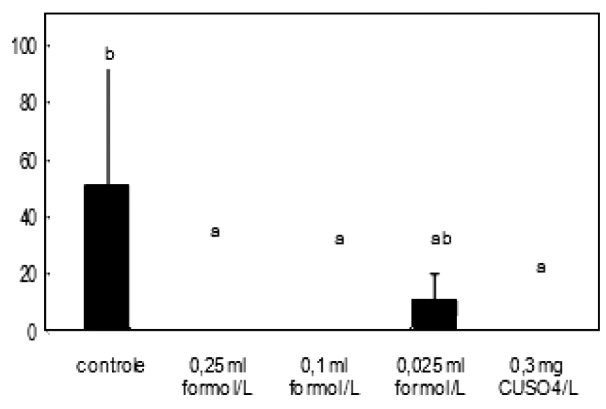

prevalëncia

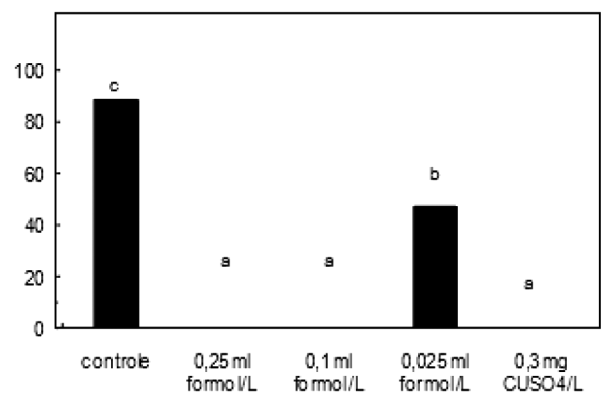

A
De posse dos dados estes foram submetidos a analise de variância (ANOVA), sendo F significativo foi realizado o teste de Tukey ( $5 \%$ de probabilidade) para comparação das médias.

\section{RESULTADOS E DISCUSSÃO}

Durante o experimento foram monitorados os parâmetros doce água, $\mathrm{pH}: 7,22 \pm 0,16$, oxigênio dissolvido: 6,87 $\pm 0,56$ $\mathrm{mg} \mathrm{L}^{-1}$ e temperatura: $29,3^{\circ} \mathrm{C} \pm 0,58$. Náo sendo esses valores prejudiciais aos peixes (Baldisseroto 2002).

No final do experimento observou-se que o T2 foi tóxico para os peixes ocasionando mortalidade de $100 \%$ do lote. De maneira geral, o manejo de troca de água pode ter provocado elevado estresse, pois nota-se mortalidade relativamente elevada, inclusive no controle. Contudo, esse é o manejo realizado comumente pelos envolvidos na comercializaçáo de peixes ornamentais quando se realiza banhos terapêuticos. No T4 observou-se a menor mortalidade (33\%).

Os peixes do controle (T1) apresentaram os maiores índices parasitológicos $(\mathrm{p}<0,05)$ : que associada ao estresse de manejo, ocasionou mortalidades de até $40 \%$. Os tratamentos T3 e T5 proporcionaram eficácia de $100 \%$ contra os parasitos, porém também foram tóxicos com mortalidades de $66,6 \%$ e $80 \%$ respectivamente. Em T4 foram obtidos resultados intermediários com eficácia de $77,7 \%$, redução do numero total de parasitas $(11,3 \pm 9)$ e da prevalência $(47,1 \%)$ e como citado anteriormente menor mortalidade. (Figura 1).
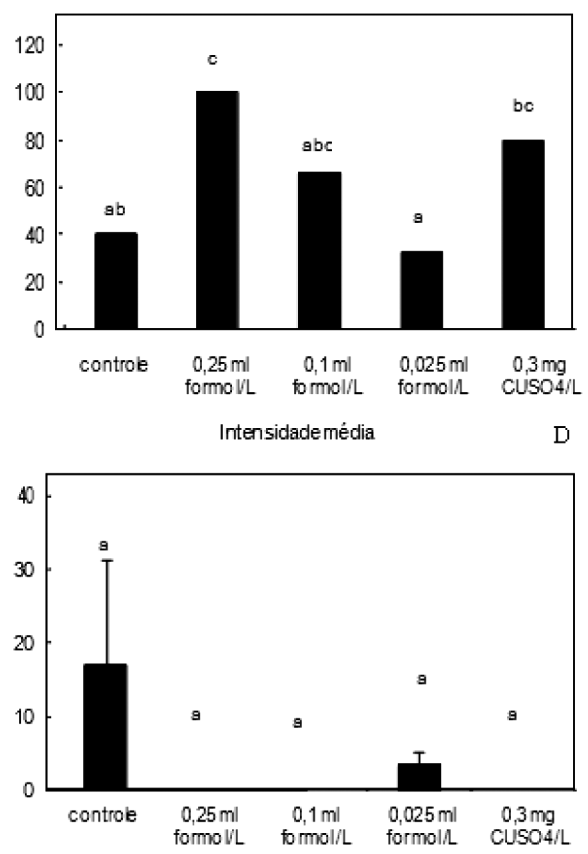

Figura 1 - Valores médios dos índices parasitológicos e da mortalidade dos peixes submetidos aos banhos com formalina e sulfato de cobre. A: número total de parasitos encontrados; B: taxa de mortalidade em \%; C: prevalência em \% e D: intensidade média de parasitos expressa em parasitos/peixe infectado. 
O T2 com $0,25 \mathrm{ml}$ de formalina $\mathrm{L}^{-1}$ foi tóxico para os peixes ocasionando mortalidade de $100 \%$, impedindo a análise da contagem diferencial de leucócitos neste tratamento. Nos demais tratamentos, após a contagem de leucócitos verificouse a ausência de basófilos e que as quantidades de leucócitos granulares PAS positivos não se alteraram em relação ao controle. Porém, pôde-se observar que houve linfocitopenia, monocitose e neutrofilia no T4 $\left(0,025 \mathrm{ml}\right.$ de formol L $\left.{ }^{-1}\right)$ em relaçáo ao controle $(\mathrm{p}<0,05)$, causado pela presença da formalina na água durante 24 horas. Inversamente no T5 $\left(0,3 \mathrm{mg}\right.$ sulfato de cobre $\left.\mathrm{L}^{-1}\right)$ e no T3 $\left(0,1 \mathrm{ml}\right.$ de formol $\left.\mathrm{L}^{-1}\right)$ houve linfocitose e monocitopenia em relação ao controle. (Figura 2).

A sobrevivência no controle e no T4 foi de 40\% e 33\%, o que indica que os peixes selvagens capturados foram sensíveis ao manejo utilizado, indicando que outras práticas de manejo devem ser adotadas pelos produtores e/ou pesquisadores que realizam tratamento nessa espécie, uma vez que o manejo por si só contribui para elevadas taxas de mortalidade.

Segundo Gandara et al. (2002) o uso da formalina é amplamente discutido na literatura e sua toxicidade varia de
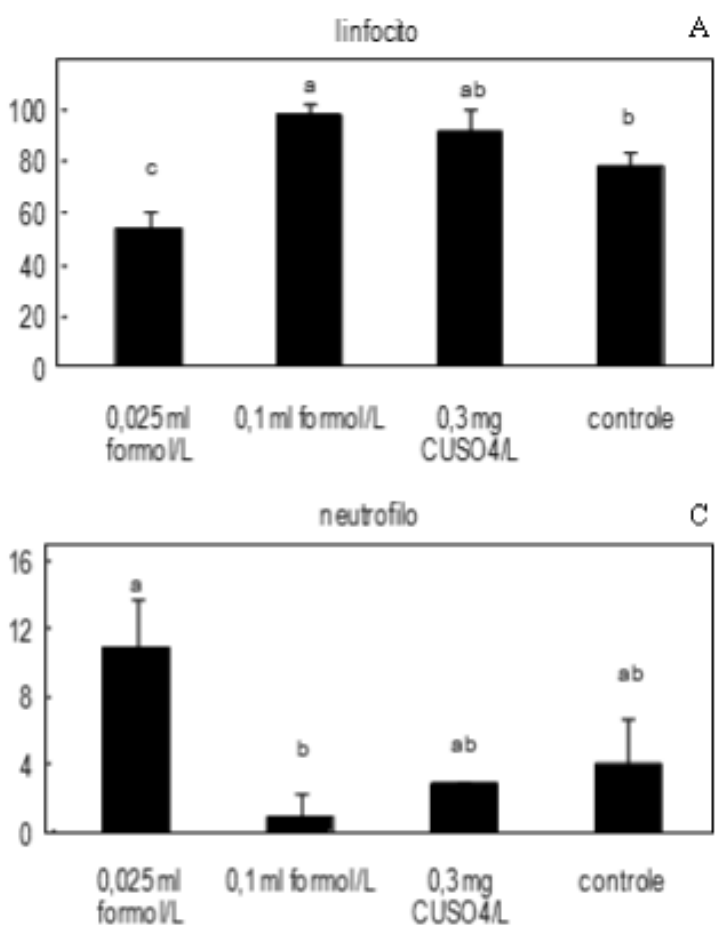

anteriormente ao tratamento é importante para a reposta ao tratamento, pois, peixes parasitados ou peixes desnutridos podem ser mais sensíveis ao tratamento com quimioterápicos e ao manejo. Além disso, em peixes pertencentes a populaçôes naturais podem apresentar comportamentos fisiológicos distintos aos peixes oriundos de regióes impactadas ou mantidos em confinamento (Santos e Tavares Dias 2010).

No peixe marinho Seriola dumerili, por exemplo, a concentração de $300 \mathrm{mg} \mathrm{L}^{-1}$ de formalina não causou mortalidades e, segundo esses mesmos autores não é restritivo o uso da formalina de forma profilática ou terapêutica para essa espécie. No presente trabalho, utilizando concentraçôes semelhantes, pôde-se observar que a formalina estressou os peixes, apresentando-se tóxico, provocando mortalidades elevadas $(100 \%$ e $66 \%)$ em banhos de curta duração como em T2 $\left(0,25 \mathrm{ml} \mathrm{L}^{-1}\right)$ e T3 $\left(0,1 \mathrm{ml} \mathrm{L}^{-1}\right)$ respectivamente.

No caso de banhos de longa duração (T4, 0,025ml formol L-1) há indícios de que os peixes exibiam um quadro decorrente de estresse em funçáo do aumento de neutrófilos e diminuição de linfócitos, resposta hematológica típico de agentes estressores (Tavares-Dias et al. 2001), apesar
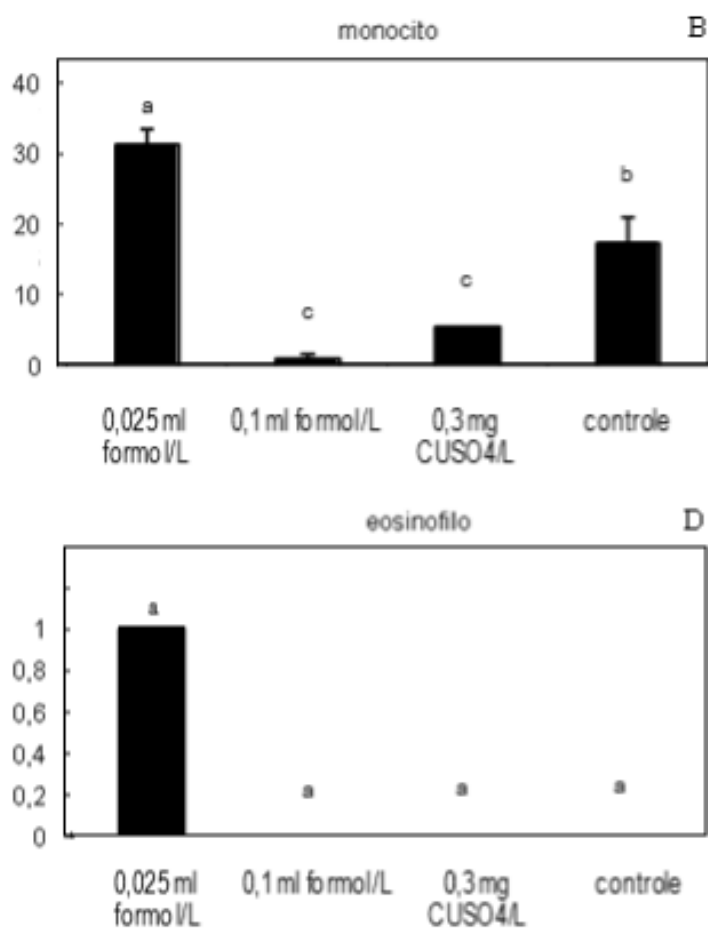

Figura 2 - Valores médios percentuais da contagem diferencial de leucócitos dos juvenis de Hemigrammus sp. submetidos ao tratamento com formalina e sulfato de cobre sendo A: linfócitos expressos em \%; B: monócitos em \%; C: neutrófilos em \% e D: eosinófilos em \%.

espécie para espécie mesmo em condições ambientais ideais de cultivo, e que podem ocorrer riscos de mortalidades quando este produto é utilizado mesmo em doses terapêuticas. Além disso, a condição sanitária e nutricional a qual o peixe está desse tratamento não apresentar mortalidade superior ao controle (33\%).

$\mathrm{O}$ estresse de manejo, associado a presença dos parasitos e a presença do quimioterápico possivelmente tenham 
desencadeado a síndrome geral de adaptação que segundo Rotlland e Tort (1997) pode ser divididas em três etapas, sendo a ultima a fase de exaustấo onde o organismo perde a homeostase e sucumbe ao agente estressor podendo levar a surtos de doenças e até mesmo a morte, o que provavelmente ocorreu pronunciadamente com os peixes submetidos aos tratamentos que receberam as maiores concentraçóes dos quimioterápicos testados (Figura. 1).

Jung et al. (2003) não observaram alteraçóes no quadro leucocitário do linguado (Paralichthys olivaceus) quando este foi submetido a banhos de uma hora com diferentes concentraçôes de formalina $\left(0,1,0,2\right.$ e $\left.0,3 \mathrm{ml} \mathrm{L}^{-1}\right)$, porém observou que o formol pode causar inibiçáo na transferência de oxigênio do sangue e danos no fígado e rim quando estes foram submetidos a banhos de curta duração. Para larvas de trairão (Hoplias lacerdae) a formalina foi considerada moderadamente tóxica com concentração letal $\left(\mathrm{CL}_{50}\right)$ em 96 horas de $0,2 \mathrm{ml} \mathrm{L}^{-1}$ e em concentraçốes de 0,1 a $0,2 \mathrm{ml} \mathrm{L}^{-1}$. O fígado destes peixes apresentaram desarranjo cordonal, fusão celular e congestáo dos sinusoides (Cruz et al., 2005). Assim, pode-se inferir que além de estressor o formol pode ter causado alteraçôes graves em órgãos dos Hemigrammus sp., comprometendo a sanidade dos peixes do presente trabalho, causando as mortalidades.

Segundo Martins (2004), o formaldeído a 37\% pode ser utilizado na forma de banho de curta duração (até 60 min) na concentraçáo de 0,15 a $0,25 \mathrm{ml} \mathrm{L}^{-1}$ e em banhos de longa duração $(24 \mathrm{~h})$ na concentraçáo de 0,010 a $0,015 \mathrm{ml}$ $\mathrm{L}^{-1}$. Porém para a espécie Hemigrammus sp capturados na natureza, o banho com formalina durante $60 \mathrm{~min}$ na mesma concentração, citada pelo autor, mostrou-se tóxica em função da mortalidade de $100 \%$. Isso pode ter ocorrido pelo fato dos peixes selvagens serem mais susceptíveis ao estresse e menos rústicos do que os de cultivo.

Segundo Rotlland et al. (1997) o Pagrus pagrus capturado na natureza apresentou aumento no nível de cortisol plasmático quando mantidos em alta densidade de estocagem. Dessa forma cuidados maiores devem ser realizados na estocagem e no tratamento de peixes nativos selvagens para exportaçáo, pois, a resposta ao estresse se torna mais acentuada e a adaptação a nova condição fica comprometida.

Segundo Cruz et al. (2005) testes preliminares são essenciais anteriormente ao tratamento definitivo com a formalina devido a sua toxicidade. Isso ocorre devido a variação muito grande em relação à concentração recomendada por diversos autores, já que há uma variação em relação a $\mathrm{CL}_{50}$ e também à uma variação da toxicidade em relação ao grau de parasitismo em que os peixes se encontram.

Quanto ao banho empregado de sulfato de cobre houve um pequeno aumento significativo nos valores de linfócitos e uma redução nos valores de neutrófilos e monócitos de
Hemigrammus sp (Figura 1). Segundo Tavares-Dias et al. (2002) consiste em resposta à alta atividade migratória e fagocíticas destas células até os locais que foram afetados pelo cobre como, brânquias, fígado e rim. Além do que a adição de metais na água pode reduzir as respostas imunes dos peixes devido ao seu efeito estressante, atuando sobre a liberaçáo de corticosteróide e catecolaminas, tornando os peixes mais susceptíveis a infecçôes.

Tavares-Dias et al. (2001) afirmaram que diversos autores demonstraram neutrofilia, em peixes estressados, acompanhando a linfocitopenia. Mazon et al. (2002) em exposição aguda ao sulfato de cobre não encontrou diminuição no número de linfócitos em Prochilodus scrofa exposto ao cobre, mas observaram aumento no de neutrófilos quando os peixes foram expostos a 96 horas em soluçáo com 20-29 $\mu \mathrm{g}$ de $\mathrm{Cu} \mathrm{L}^{-1}$. Além disso, não observou alteraçóes nos valores de monócitos corroborando com Fujimoto et al. (2009). Esses autores não verificaram diferença nos valores de monócito em peixes parasitados e não parasitados, contrariamente ao observado no presente estudo.

Essas variaçóes podem ocorrer devido a concentraçáo testada e o grau de dano que o cobre causa aos órgáos, pois, a alteraçáo nos valores de leucócitos varia de acordo com a migração das células do vaso para o tecido lesado, além disso uma lesão em órgãos hematopoéticos também pode alterar esse quadro (Mazon et al., 2002; Tavares-Dias et al., 2002).

Tavares-Dias et al. (2002) estudaram a hematologia de pacus (Piaractus mesopotamicus) submetidos a banhos de sulfato de cobre por 24 horas em duas aplicaçóes intermitentes e observaram que 24 horas depois do último banho houve diminuição do número de neutrófilos, quando expostos a concentração de $0,5 \mathrm{mg} \mathrm{L}^{-1}$, porém os autores relataram diminuição no número de linfócitos aos 8 dias após a exposição ao produto.

\section{CONCLUSÕES}

O sulfato de cobre e a formalina, reduzem a carga parasitária, mas foram tóxicos nas maiores concentraçōes. Banhos de 24 horas com formalina seriam mais indicados, porém outras concentraçóes devem ser testadas para peixes nativos e selvagens como o Hemmigramus sp. A duração dos banhos influenciou na proporção dos leucócitos sendo que mesmo em dosagens baixas recomendadas a formalina parece ter comprometido a saúde dos peixes.

\section{AGRADECIMENTOS}

A Poytara pelo fornecimento da ração para a realização do experimento e a CAPES pela concessáo da bolsa de doutorado ao terceiro autor. 


\section{BIBLIOGRAFIA CITADA}

Araújo, L.D.; Chagas, E.C.; Gomes; Brandão, F.R. 2004. Efeito de banhos terapêuticos com formalina sobre indicadores de estresse em tambaqui. Pesquisa Agropecuária Brasileira, 39 : 217-221.

Baldisserotto, B. 2002. Fisiologia de Peixes Aplicada à Piscicultura. Ed. UFSM, Santa Maria. 212 pp.

Bassleer, G. 2009. The new illustrated guide tofish diseases. Bassleer Biofish, Westmeerbeek, Belgium. 232pp.

Goulding, M. 1980. The fishes and the forest. Explorations in Amazonian natural history. University of California Press, Berkeley, CA, USA. 280 pp.

Brandão-Gonçalves, L.; Oliveira, S.A.; Lima-Junior, S.E. 2010. Hábitos alimentares da ictiofauna do córrego Franco, Mato Grosso do Sul, Brasil. Biota Neotropica, 10: 21-30.

Bush, A.O.; Lafferty, K.D.; Lotz, J.M.; Shostak, A.W. 2009. Parasitology meets ecology on its own terms: Margolis et al. revisited. Journal of Parasitology, 83 : 575-583.

Carraschi, S.P.; Cubo, P.; Schiavetti, B.L.; Shiogiri, N.S.; Cruz, C. Pitelli, R.A. 2011. Efeitos tóxicos de surfactantes fitossanitários para o peixe mato grosso (Hyphessobrycon eques). Acta Scientiarum. Biological Sciences, 33 : 191-196.

Casatti, L.; Mendes, H.F.; Ferreira, K.M. 2003. Aquatic macrophytes as feeding site for small ishes in the Rosana reservoir, Paranapanema river, southeastern Brazil. Brazilian Journal of Biology. 63 : 213-222.

Cruz, C.; Fujimoto, R.Y.; Luz, R.K.; Portella, M.C.; Martins, M.L. 2005. Toxicidade aguda e histopatologia do fígado de larvas de trairão (Hoplias lacerdae) expostas à solução aquosa de formaldeído a 10\%. Pesticidas: Revista de Ecotoxicologia e Meio Ambiente. $15: 21-28$.

Fujimoto, R.Y.; Santana, C.A.; Carvalho, W.L.C.; Diniz, D,G.; Barros, Z.M.N.; Varella, J. E. A.; Guimarães, M. D. F. 2009. Hematologia e parasitas metazoários de camurim (Centropomus Undecimalis, bloch, 1792) na região Bragantina, Bragança-Pará. Boletim do Instituto de Pesca. 35 : 441-450.

Gándara, F.; Jover, M.; García-Gómez, A. (2002). Efecto del tratamiento con formol sobre el consumo de oxígeno de juveniles de seriola mediterránea Seriola dumerili (Risso, 1810). Bol. Inst. Esp. Oceanogr. 377-383

Garcia, F; Fujimoto, R.Y.; Martins, M. L.; Moraes, F. R. 2003. Parasitismo de Xiphophorus spp. por Urocleidoides sp. e sua relação com os parâmetros hídricos, Boletim do Instituto de Pesca. 29 : 123-131.

Jung, S.H.; Sim, D.S.; Park, M.; Jo, Q.; Kim, Y. 2003. Effects of formalin on haematological and blood chemistry in olive flounder, Paralichthys olivaceus. (Temminck et Schlegel). Aquaculture Research. 34 : 1269-1275.
Martins, M.L., 2004. Cuidados básicos e alternativas no tratamento de enfermidades de peixes na aqüicultura brasileira, p.357-70. In: RANZANI-PAIVA, M.J., TAKEMOTO, R.M., LIZAMA, M.A.P. Sanidade de Organismos Aquáticos. São Paulo: Editora Varela.

Mazon, A. F.; Monteiro, E. A. S.; Pinheiro, G. H. D.; Fernandes, M. N. 2002. Hematological and physiological changes induced by short-term exposure to copper in the freshwater fish, Prochilodus scrofa. Brazilian Journal of Biology. 62: 621-631.

Rotlland, J.; Pavlidis, M.; Kentouri, M.; Abad, M.E.; Tort, L. 1997. Non-specific immune reponses in the red progy pagrus pagrus after crowding stress. Aquaculture. $156: 279-90$.

Rotlland, J.; Tort, L. 1997. Cortisol and glucose response after acute stress by net handling in the sparid red porgy previously to subject crowding stress. Journal Fish Biology. 51: 21-28.

Santos, R. B. S.; Tavares-Dias, M. 2010. Células sanguíneas e resposta hematológica de Oxydoras niger (pisces, doradidae) oriundos da bacia do médio rio solimóes, Boletim do Instituto de Pesca. 36 : 283-292.

Tavares-Dias, M.; Sandrim, E.F.S.; Moraes, F.R.; Carneiro, P.C.F. 2001. Physiological responses of "tambaqui" Colossoma macropomum (CHARACIDAE) to acute stress. Boletim do Instituto de Pesca. 27 : 43-48.

Tavares-Dias, M.; Martins, M. L.; Schalch, S. H. C.; Onaka, E. M.; Quintana, C. I. F.; Moraes, J. R.E.; Moraes F. R. 2002. Alterações hematológicas e histopatológicas em pacu, Piaractus mesopotamicus Holmberg, (1887) (Osteichthyes, Characidae), tratado com sulfato de cobre (CuSO4). Acta Scientiarum. Animal Science. 24 : 547-554.

Torres, M.F.; Giarizzo, T.; Carvalho, J.R.Jr. Diagnóstico, Tendência, Análise e Políticas Públicas para o Desenvolvimento da Pesca Ornamental no Estado do Pará. Belém: SEPAq, 2008. 41p.

Vargas, L. 2004. Efeito da Vitamina C, da Vitamina E, do Cloreto de Sódio e da Formalina na Ocorrência de Ectoparasitas em Tilápias do Nilo (Oreochromis niloticus). p. 371-382. In: Ranzani-Paiva, M.J.; Takemoto, R.M.; Lizama, M.A.P. Sanidade de Organismos Aquáticos. São Paulo: Editora Varela.

Recebido em: 13/10/2011

Aceito em: 04/05/2012 